\title{
A Q-switched Erbium Doped Fibre Laser utilising a novel large mode area fibre
}

Gareth P. Lees, D. Taverner, D.J. Richardson, L. Dong and Trevor P. Newson

Paper reference no: \#1396

\author{
Optoelectronics Research Centre, University of Southampton \\ Southampton, SO17 1BJ. United Kingdom \\ Tel. +441703593172 Fax. +441703593149
}

E-MailGPL@ORC.SOTON.AC.UK

\begin{abstract}
A High Power Q-switched Erbium doped fibre laser has been demonstrated using a novel, large mode area, single transverse mode fibre. Peak Powers in excess of $4 \mathrm{~kW}$, and pulse widths of $10 \mathrm{~ns}$ have been reported at a repetition rate of $500 \mathrm{~Hz}$. These results represent the highest peak powers obtained from an actively Q-switched Erbium Doped Fibre Laser.
\end{abstract}


Introduction: Q-switched fibre lasers were first developed in 1986 [1], and the advances achieved since then have been largely due to improvements in acousto-optic modulator design, advances in pump laser technology [2], the use of more efficient modulators [3,4], and advantages gained with co-doped systems such as Er/Yb [5]. In this paper we report advances in Q-switched laser performance by focusing on a novel fibre geometry which improves the energy storage within the fibre. Two methods can be applied to increase the energy stored in the fibre per unit length. One can either increase the Erbium concentration or increase the core area. Increasing the Erbium concentration eventually leads to clustering of the Erbium ions which decreases the efficiency of the fibre by a process of co-operative up-conversion. Recent numerical modeling [7] has indicated that for maximum energy extraction a large mode field area (LMA) is beneficial. This method of increasing the energy storage of Erbium doped fibre has been recently demonstrated in a large mode area Erbium doped fibre amplifier, pulse energies of $158 \mu \mathrm{J}$ have been produced using a multi-stage amplifier chain [6]. It is this novel fibre design which we have applied to Q-switched Erbium doped fibre lasers to increase the pulse output energy.

The technique of increasing the core area is limited by the requirement that the fibre remains single mode at the signal wavelength. Single mode operation is maintained by decreasing the N.A. of the fibre, which is achieved by reducing the refractive index difference $\Delta \mathrm{n}$, between the fibre core and cladding.

The novel fibre developed in this study has a step-index profile with a core radius of $7.3 \mu \mathrm{m}$, and an N.A. of 0.08 , the resulting mode field radius is therefore $8.04 \mu \mathrm{m}$. This radius corresponds to a mode field area of $208 \mu \mathrm{m}^{2}$, compared to the $50 \mu \mathrm{m}^{2}$ area of a typical Erbium doped fibre. The fibre had an Erbium concentration of approximately 4000ppm.

The mode field diameters for both conventional and large mode area Erbium fibres were measured and are shown in figure 1 . These results were obtained using an infrared vidicon camera to view the far field patterns on a screen positioned $220 \mathrm{~mm}$ from the fibre end. The measured far field diameters (full width half height) for the conventional and LMA fibres were $67.7 \mathrm{~mm}$ and $35.5 \mathrm{~mm}$ respectively. A simple trigonometric approach yields values for the numerical aperture of 0.08 and 0.15 for the LMA and conventional Erbium fibres respectively, this is in agreement with 
preform measurements. It is also clear from figure 1 that the large mode area fibre still propagates a single transverse mode.

Decreasing the refractive index difference, $\Delta \mathrm{n}$, between core and cladding leads to the fibre becoming more sensitive to bend loss, however, this is not a problem for Qswitched lasers which typically use less than 1 metre of fibre.

The low N.A. of the fibre and the increased mode field area has two principal advantages. Firstly, for a given absorbed pump power, increasing the mode field area reduces the amplified spontaneous emission (ASE). The reduction of the ASE allows an increase in stored energy to be obtained [7]. Secondly, the increased mode area also reduces non-linear Raman effects which at high powers can cause the pulse energy to be shifted to higher wavelengths.

This novel fibre enables short high power pulses to be obtained, suitable for applications of eyesafe laser range finding, OTDR, remote sensing and free space communications.

Experiment: The experimental arrangement is shown in figure 2. An Argon pumped Ti-Sapphire is used as the pump source with up to 1.2 Watts of output power at 980 $\mathrm{nm}$. This pump was launched into the large mode area fibre through a 980/1530nm dichroic filter, which was used to obtain the output from the laser. The far end of the fibre was polished at an angle of $16^{\circ}$ to prevent the fibre lasing from the $4 \%$ Fresnel reflection. The length of fibre used in the experiment was $63 \mathrm{cms}$ and was optimised for the available launched pump power. The output fluorescence was then focused through an acousto-optic modulator (AOM) onto a mirror which had a $99 \%$ reflectivity at 1530-1550nm. Zero order operation of the AOM produced the best results due to the relatively low diffraction efficiency available from the AOM (40-50\%).

Using this configuration peak powers in excess of $4 \mathrm{~kW}$ with associated pulse widths of $11 \mathrm{~ns}$ were obtained at a repetition rate of $<1 \mathrm{kHz}$. The variation of peak power and pulse width with repetition rate is shown in figure 3 and is typical of a Q-switched Erbium laser. The fall off of pulse energy with higher repetition rates is due to the finite recovery time of the population inversion, which is directly related to the lifetime of the metastable level, typically $10-12 \mathrm{~ms}$ for Erbium. This lifetime is reduced to an effective 
lifetime of $1 \mathrm{~ms}$ due to the presence of amplified spontaneous emission (ASE), which depleted the upper laser level. Figure 4 shows the variation of pulse energy with estimated launched power, assuming a 50\% launch efficiency. The increase in pulse energy with launched pump power displays a saturation effect at high pump powers. This saturation can be explained by the increase in ASE power for high pump powers which clamps the available gain and subsequently the pulse energy. For estimated launched pump powers of $600 \mathrm{~mW}$ the measured pulse energy is $50 \mu \mathrm{J}$.

Conclusion: We report a large increase in pulse energy from a Q-switched Erbium doped fibre laser using a specially fabricated low N.A. fibre. The continuing optimisation of the large mode area fibre with respect to dopant concentration and core radius should result in pulse energies in excess of $100 \mu \mathrm{J}$ from portable MOPA pumped systems.

Acknowledgements: This work is supported by a UK Engineering and Physical Sciences Research council (EPSRC) CASE award in collaboration with York Sensors Ltd, Chandlers Ford. 
References:

[1] Mears, R.J., Reekie, L., Poole, S.B. and Payne, D.N.: "Low threshold, tunable CW and Q-switched fibre laser operating at $1.55 \mu \mathrm{m} . ”$, Electronics Letters, Vol.22, pp.159-160, Jan. 1986

[2] Seguin, F. and Oleskevich, T.: "Diode Pumped Q-switched fibre laser", Optical Engineering, Vol. 32, No. 9, Sep. 1993

[3] Lees, G.P. and Newson, T.P.: "Diode Pumped high power simultaneously Qswitched and self mode-locked Erbium doped Fibre Laser", Electronics Letters, Vol. 32, No. 4, Feb. 1996

[4] Chandonnet, A. and Larose, G.: "High Power Q-switched Erbium Fibre laser using an all-fibre intensity modulator", Optical Engineering, Vol 32, No. 9, Sep. 1993

[5] Lees, G.P., Hartog, A., Leach, A. and Newson, T.P.: “980nm Diode Pumped Er/Yb doped Q-switched Fibre Laser", Electronics Letters, Vol 31, No. 21, Oct. 1995

[6] Taverner, D., Richardson, D.J., Dong, L., Caplen, J.E., Williams, K., Penty,R.V.: “158 $\mu$ J Pulses from a single Transverse Mode, Large Mode-Area EDFA", Optics Letters, In press.

[7] Nilsson, J. and Jaskorzynska, B.: "Modelling and optimization of low repetition rate high energy pulse amplification in CW pumped Erbium doped fibre amplifiers", Optics Letters, Vol.18, No.24, Dec.1993 
Figure Captions:

Figure 1 - This shows the far field mode patterns for both the conventional and large mode area fibres.

Figure 2 - Experimental arrangement for the Q-switched Fibre laser

Figure 3 - Experimental results showing the variation of peak power and pulse width with repetition rate

Figure 4 - Experimental results showing the variation of Pulse Energy with launched pump power 


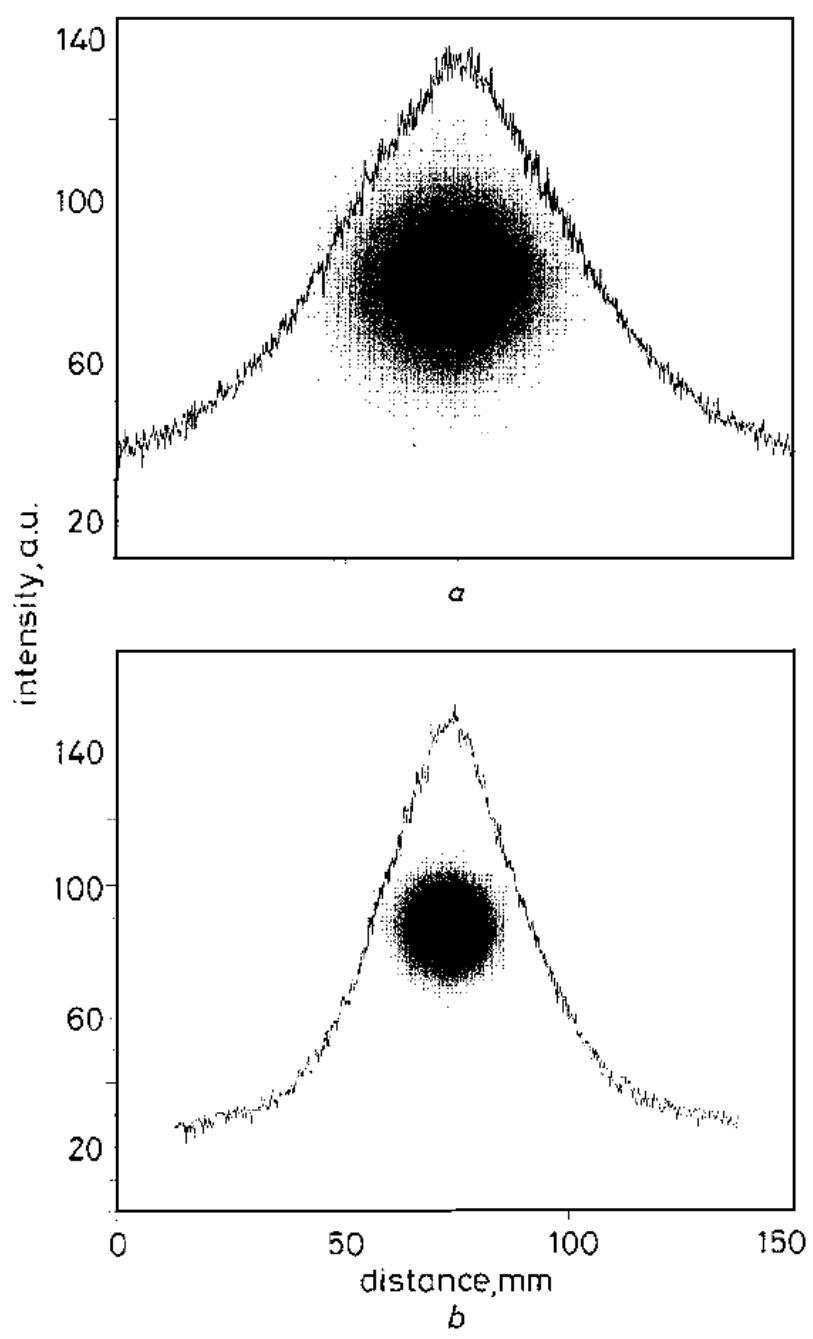

Figure 1

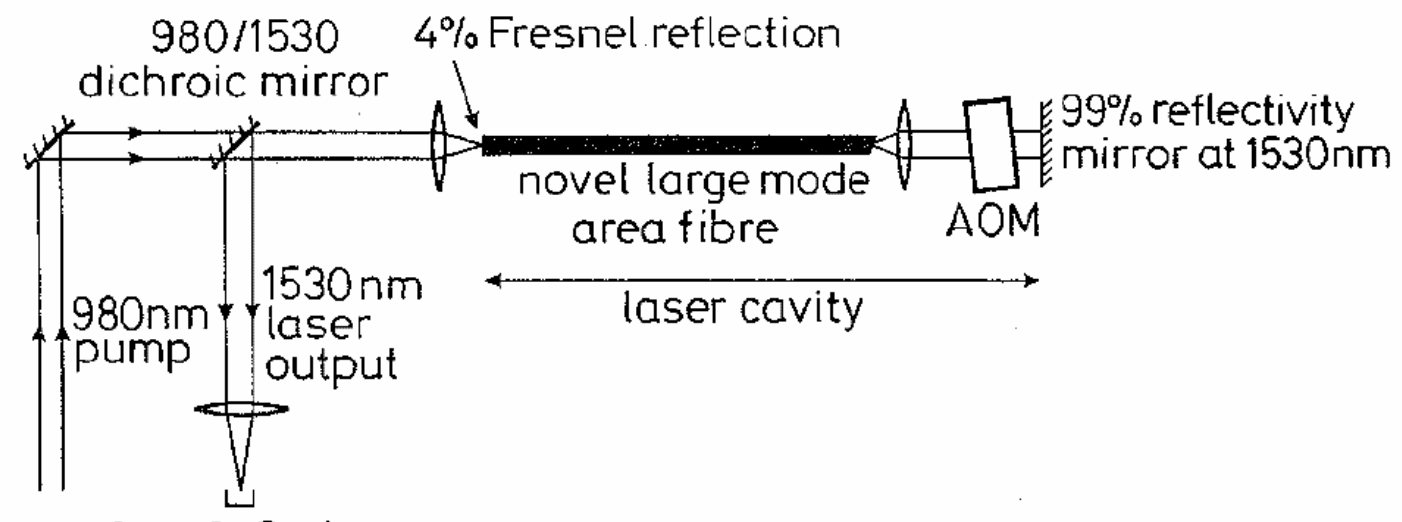

fast InGaAs

detector

Figure 2 


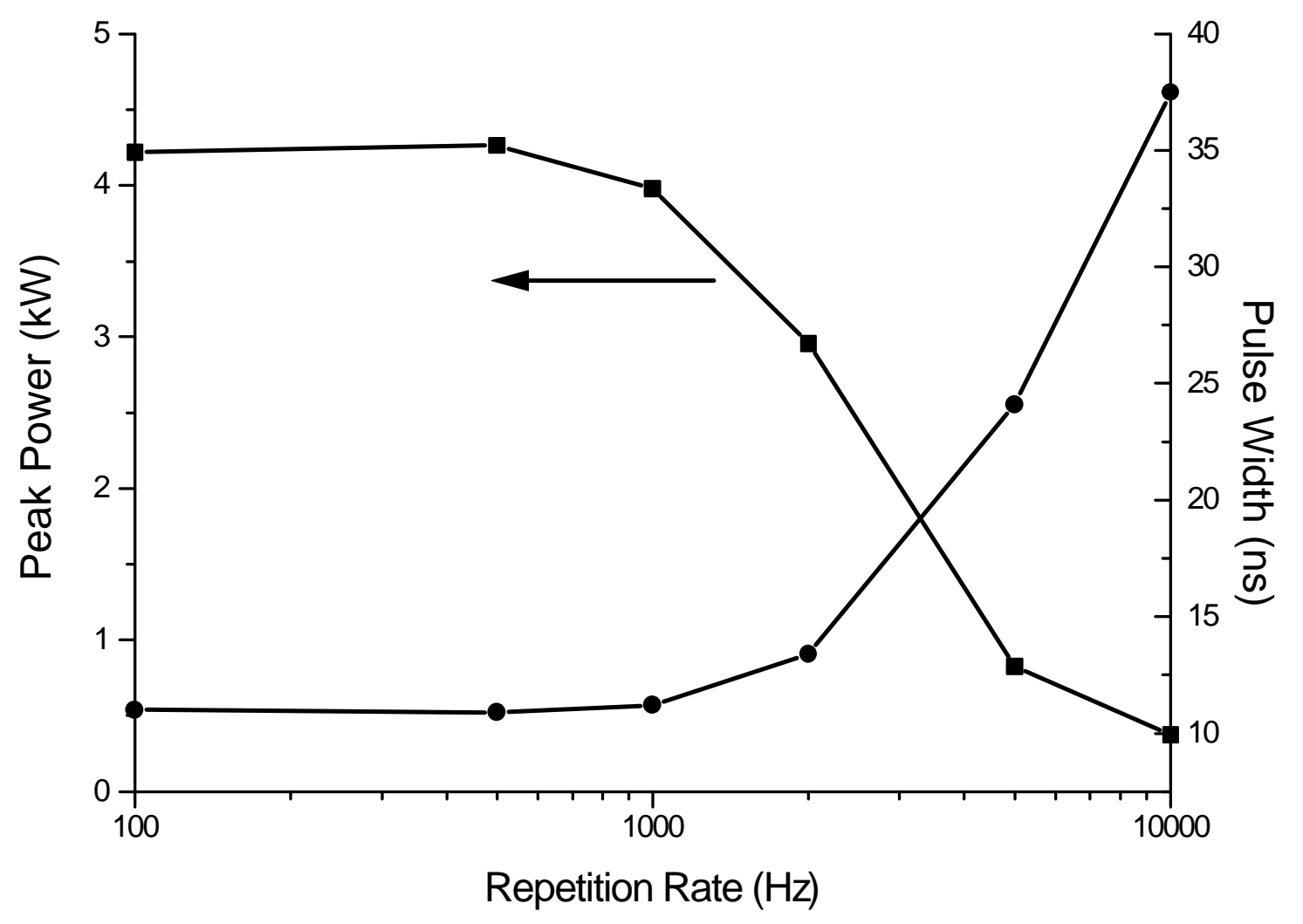

Figure 3 


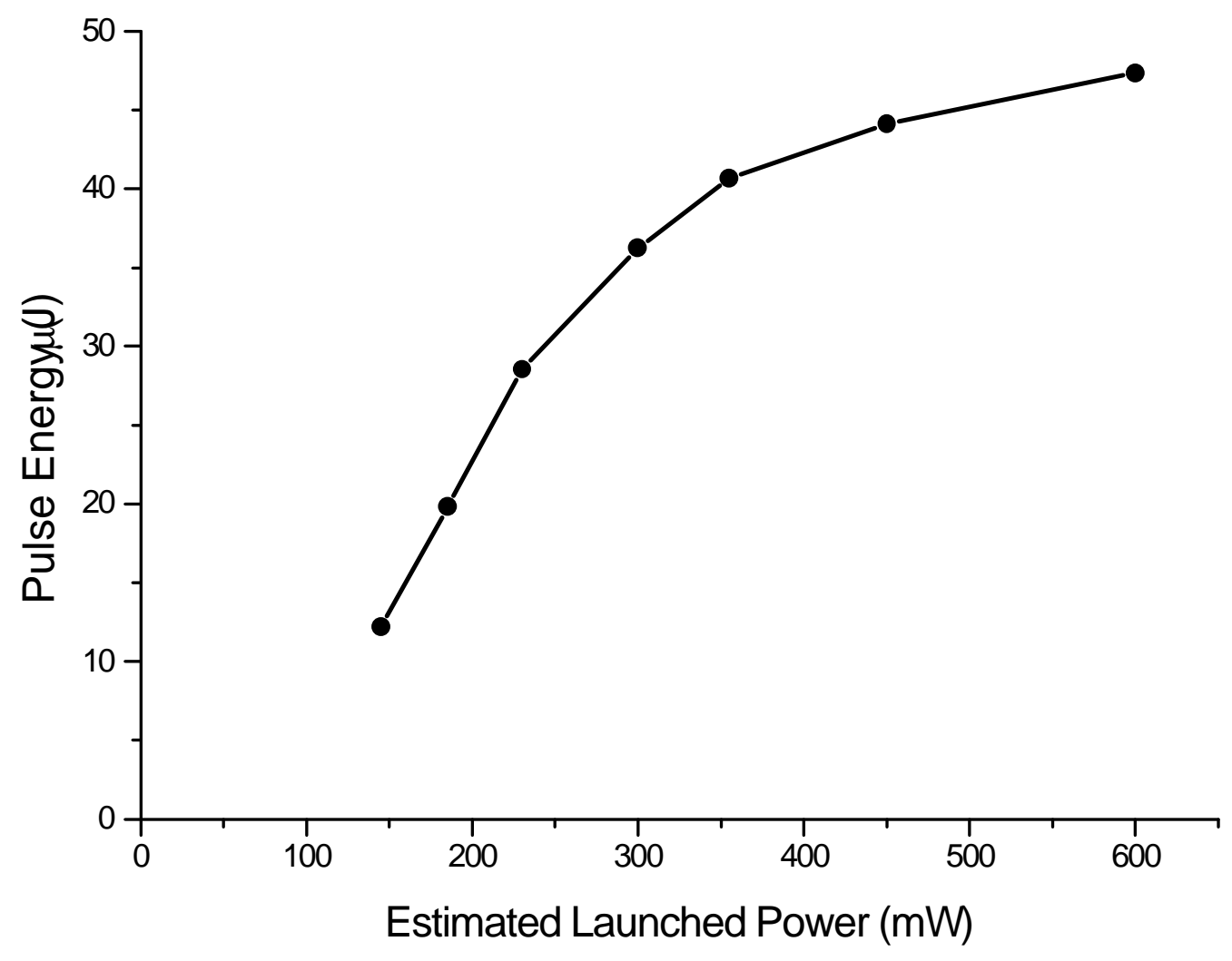

Figure 4 